\title{
PREDICTORS OF ATRIAL FIBRILLATION RECURRENCE AFTER RADIOFREQUENCY ABLATION IN PATIENTS WITH CHRONIC HEART FAILURE
}

DOI: $10.36740 /$ WLek202108113

\author{
Tetiana V. Zolotarova', Mariia S. Brynza' ${ }^{1}$, Dmytro Y. Volkov' ${ }^{2}$, Maryna I. Shevchuk' ${ }^{1}$, Oleksandr V. Bilchenko' \\ 'V. N. KARAZIN KHARKIV NATIONAL UNIVERSITY, KHARKIV, UKRAINE \\ ${ }^{2}$ V. T. ZAYCEV INSTITUTE OF GENERAL AND URGENT SURGERY NATIONAL ACADEMY OF MEDICAL SCIENCES OF UKRAINE, KHARKIV, UKRAINE
}

\begin{abstract}
The aim: To study the predictive power of demographic, hemodynamic and electrocardiographic factors for atrial fibrillation recurrence after radiofrequency ablation (RFA) in patients with chronic heart failure.

Materials and methods: Study included 120 patients, aged 59,80 10,08 years old with chronic heart failure with preserved left ventricular ejection fraction who were undergo RFA due to atrial fibrillation (AF). A standard 12-lead electrocardiogram (ECG) was registered. Before the procedure the standard echocardiographic parameters were obtained. After 12 months, patients were divided into 2 groups: non-recurrence group and recurrence group.

Results: As a result of prospective follow-up within 12 months AF recurrences were observed in 32 patients (27\%), 88 patients remained non-recurrent (73\%). The left atrium (LA) diameter and aortic root diameter were lager in the recurrence group ( $4,59 \pm 0,45 \mathrm{vs.} 4,08 \pm 0,61 \mathrm{~cm}, p<0,001 ; 3,37 \pm 0,60 \mathrm{vs} .2,80 \pm 0,67 \mathrm{~cm}, p<0,001)$. The AF recurrence group before RFA had a significantly longer QTc interval than the non-recurrence group $(387,23 \pm 2,31 \mathrm{vs}$. $341,22 \pm 8,91 \mathrm{~ms}, \mathrm{p}<0,010)$. ROC curve analysis revealed LA diameter the most sensitive factor for AF recurrence after RFA.

Conclusions: QTc duration before radiofrequency ablation and its prolongation after intervention are independent predictors of atrial fibrillation recurrence; left atrium diameter before ablation is a highly sensitive predictor in patients with chronic heart failure with preserved left ventricular ejection fraction.
\end{abstract}

KEY WORDS: atrial fibrillation, recurrence, heart failure, radiofrequency ablation

Wiad Lek. 2021;74(8):1850-1855

\section{INTRODUCTION}

Atrial fibrillation (AF) is the most common type of cardiac arrhythmias and is associated with the more than 3-fold higher risk of development of heart failure (HF) and a 2-fold higher risk of mortality [1].

Randomized controlled trials and meta-analyzes have shown high efficacy of radiofrequency ablation (RFA) for both initial and secondary strategies after unsuccessful medical therapy (MT) to maintain sinus rhythm (SR) and improve functional and morphological capacity of cardiac activity, improvement of quality of life; in patients with chronic HF RFA was associated with a significant relative reduction in the risk of overall mortality, AF recurrence, and hospitalization for cardiac pathology (compared with MT) [1-3].

The main problem of RFA remains high risk of AF recurrence (up to $45 \%$ within 6-12 months after intervention) [4], especially, in the late period, which is associated with the unfavorable prognosis.

Multiple studies have focused on searching of available predictors of AF recurrence after RFA to increase the effectiveness of the RFA in patients with different cardiovascular comorbidities $[5,6]$.

Over the last decade, several studies have identified several major factors such as age, duration of AF, duration of
AF paroxysm cycle, left and right atrium size (LA and RA), left ventricular ejection fraction (LVEF), as predictors of AF recurrence after the first successful RFA [6-8].

Nevertheless, these studies are controversial and need further study.

\section{THE AIM}

Study the predictive power of demographic, hemodynamic and electrocardiographic factors for atrial fibrillation recurrence after radiofrequency ablation in patients with chronic heart failure.

\section{MATERIALS AND METHODS}

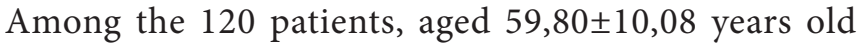
with chronic HF with preserved LVEF New York Heart Association (NYHA) functional class between I and III who were undergo RFA due to AF, 50 (42\%) - female, 70 (58\%) -male. A diagnosis of HF with preserved LVEF was made in accordance with the current guidelines [9] as a $\mathrm{LVEF} \geq 50 \%$, presence of typical symptoms and/or signs, $\mathrm{LV}$ diastolic dysfunction and/or relevant structural heart disease fulfilled within 6 months prior to AF ablation. The 
type of AF include: paroxysmal $(n=60)$, persistent $(n=54)$, long-standing persistent $(n=6)$. The strategy of RFA included bilateral pulmonic vein isolation (PVI) for paroxysmal $\mathrm{AF}$ and PVI plus linear ablation of linear lesions, mitral isthmus and tricuspid isthmus for persistent $\mathrm{AF}$ and associated typical atrial flutter (AFL).

Baseline variables comprised demographic and clinical data, medical history (coronary heart disease, hypertension, diabetes, and previous stroke).

A standard 12-lead electrocardiogram (ECG) was registered 1 day prior to the procedure in all patients. QT interval measurement was done in lead II or V5 where it was best seen. The QTc interval was calculated by using Bazett's formula in patients with SR (31\%) where heart rate $(\mathrm{HR})$ was between 60 to 100 beats per minute $(\mathrm{QTc}=$ $\left.\mathrm{QT} /\left(\mathrm{RR}^{\wedge} 0,5\right)\right)$. If a patient had AF $(69 \%)$ or HR differed from 60 to 100 beats per minute, the QTc was calculated by Framingham formula where QT interval measured from 3 consecutive cardiac cycles and then a mean value was used for analysis.

1 day before the procedure, all patients underwent transthoracic echocardiography (TTE). The standard echocardiographic parameters were obtained by utilizing the techniques recommended by current guidelines [10], especially: LVEF, left ventricle end-diastolic diameter and volume (LVED and LVEDV), left ventricle end-systolic diameter and volume (LVES and LVESV), left and right atrium diameter (LA and RA, respectively), diastolic right ventricle diameter (RVD), diameter of aortic root, mean pulmonary artery pressure.

The MT for AF was administered in accordance with the guidelines that were available at the time of the research.

After the RFA, all patients received antiarrhythmic drugs if there were no contraindications. The drugs were discontinued if no recurrent atrial tachyarrhythmia was detected after 3 months after procedure. All patients had anticoagulant treatment for at least 3 months after the RFA and MT for concomitant chronic HF [11].
After 12 months, patients were divided into 2 groups: non-recurrence group and recurrence group. An episode of supraventricular arrhythmia or AF lasting longer than 30 seconds was considered as recurrence by the ECG for the past 12 months after RFA arrhythmia.

The obtained data after the formation of the database was processed in Microsoft Excel, SPSS 17.0. For statistical evaluation of the results parametric criteria were used and nonparametric criteria. Reliability of the differences between groups was evaluated using the nonparametric Mann-Whitney U Test. The results were considered reliable at the significance levels $\mathrm{p}<0,05$. Spearman's correlation analysis, stepwise discriminant analysis, regression analysis, Receiver Operating Characteristic (ROC) - analysis were done.

\section{RESULTS}

As a result of prospective follow-up within 12 months AF recurrences were observed in 32 patients (27\%), 88 patients remained non-recurrent (73\%). The baseline characteristics of the study population depending on the group were shown in Table I. Elder patients prevailed in the recurrence group $(63,25 \pm 10,80$ versus (vs.) $58,66 \pm 9,52 ; \mathrm{p}<0,010)$. The duration of QRS complex was normal in both groups, but longer in the recurrence group $(96,79 \pm 23,62$ vs. $90,24 \pm 14,78 \mathrm{~ms}, \mathrm{p}<0,010)$. The LA diameter and aortic root diameter were lager in the recurrence group $(4,59 \pm 0,45$ vs. $4,08 \pm 0,61 \mathrm{~cm}, \mathrm{p}<0,001$; $3,37 \pm 0,60$ vs. $2,80 \pm 0,67 \mathrm{~cm}, \mathrm{p}<0,001)$. The AF recurrence group had a significantly higher mean pulmonary artery pressure $(32,86 \pm 9,67$ vs. $25,15 \pm 9,73 \mathrm{mmHg}, \mathrm{p}<0,010)$. The was slight significant difference in LVEF: in the recurrence group it was lower $(59,08 \pm 9,60$ vs. $63,36 \pm 9,10 \%, p=0,051)$.

There was no significant difference in gender, duration of AF, history of hypertension, body mass index (BMI), history of coronary heart disease, history of diabetes, or history of stroke/ transient ischemic attack of the AF recurrence and non-AF recurrence groups. There were no significant differences in

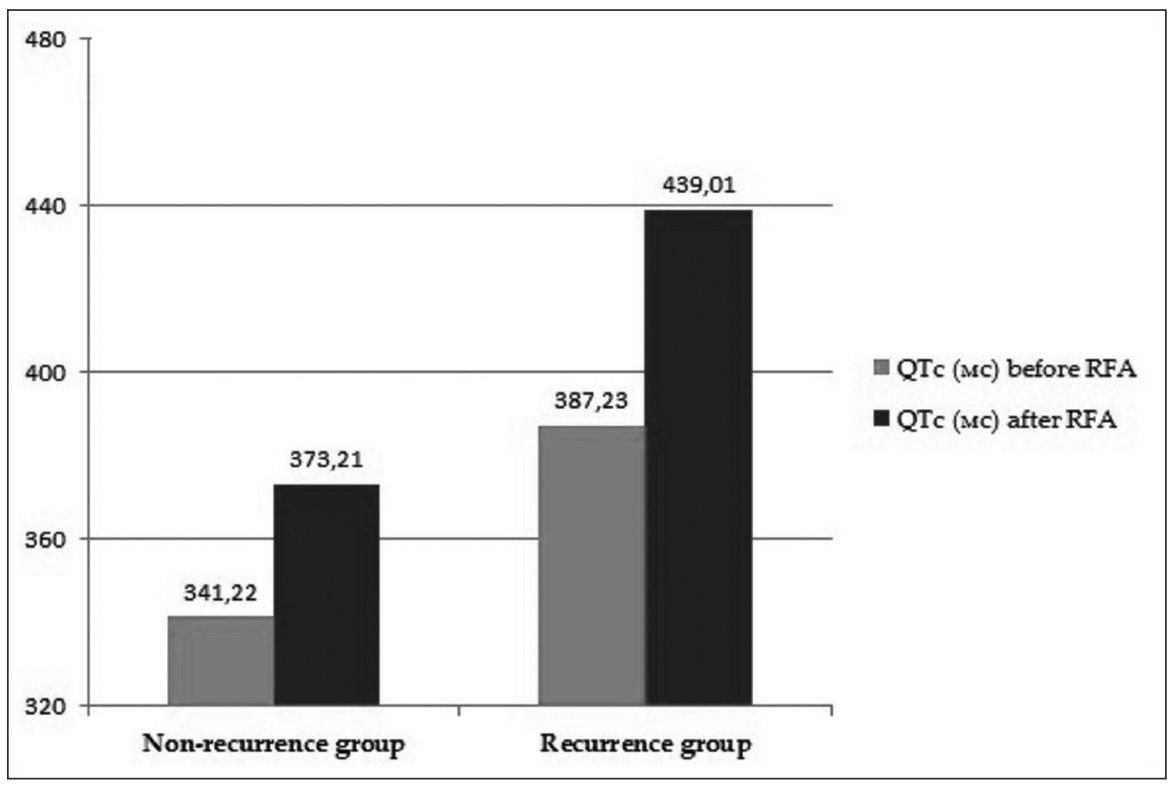

Fig. 1. Distribution of QTc in patients with/without recurrence after atrial fibrillation ablation 
Table I. Baseline characteristics of the study population

\begin{tabular}{|c|c|c|c|}
\hline Characteristics & $\begin{array}{l}\text { Non-recurrence group } \\
\qquad \begin{array}{c}n=88(73 \%) \\
M \pm s d / \%\end{array}\end{array}$ & $\begin{array}{l}\text { Recurrence group } \\
\begin{array}{c}n=32(27 \%) \\
M \pm s d / \%\end{array}\end{array}$ & P-value \\
\hline Age (years) & $58,66 \pm 9,52$ & $63,25 \pm 10,80$ & $<0,010$ \\
\hline Sex (male/female) & $61 / 39$ & $50 / 50$ & 0,082 \\
\hline AF duration (years) & $4,58 \pm 12,54$ & $4,75 \pm 2,33$ & 0,861 \\
\hline Body mass index (kg/m2) & $26,80 \pm 5,56$ & $26,6 \pm 4,83$ & 0,836 \\
\hline Coronary artery disease & 11,36 & 31,25 & 0,192 \\
\hline Hypertension & 84,09 & 84,37 & 0,374 \\
\hline Diabetes mellitus & 17,10 & 18,75 & 0,564 \\
\hline Stroke/Transient Ischemic Attack & 5,75 & 6,25 & 0,802 \\
\hline $\mathrm{HR}$, bpm & $81,06 \pm 26,74$ & $80,81 \pm 22,35$ & 0,276 \\
\hline $\mathrm{PR}(\mathrm{Q}), \mathrm{ms}$ & $171,1 \pm 16,94$ & $173,4 \pm 16,55$ & 0,860 \\
\hline $\mathrm{QRS}, \mathrm{ms}$ & $90,24 \pm 14,78$ & $96,79 \pm 23,62$ & $<0,010$ \\
\hline QTc, ms & $341,22 \pm 8,91$ & $387,23 \pm 2,31$ & $<0,010$ \\
\hline LVEF, \% & $63,36 \pm 9,10$ & $59,08 \pm 9,60$ & 0,051 \\
\hline LVED, cm & $5,03 \pm 0,73$ & $4,98 \pm 0,56$ & 0,548 \\
\hline LVES, cm & $3,23 \pm 0,75$ & $3,33 \pm 0,54$ & 0,915 \\
\hline LVEDV, ml & $101,74 \pm 63,77$ & $98,36 \pm 57,86$ & 0,542 \\
\hline LVESV, ml & $37,74 \pm 28,68$ & $36,47 \pm 25,85$ & 0,915 \\
\hline LA diameter, $\mathrm{cm}$ & $4,08 \pm 0,61$ & $4,59 \pm 0,45$ & $<0,001$ \\
\hline RA diameter, $\mathrm{cm}$ & $4,20 \pm 0,68$ & $4,24 \pm 0,46$ & 0,486 \\
\hline $\mathrm{RVD}, \mathrm{cm}$ & $2,75 \pm 0,52$ & $2,83 \pm 0,50$ & 0,542 \\
\hline Aortic root diameter, $\mathrm{cm}$ & $2,80 \pm 0,67$ & $3,37 \pm 0,60$ & $<0,001$ \\
\hline Mean pulmonary artery pressure, $\mathrm{mmHg}$ & $25,15 \pm 9,73$ & $32,86 \pm 9,67$ & $<0,010$ \\
\hline
\end{tabular}

*Numbers are presented as $n(\%)$ or mean (M) \pm standard deviation (sd).

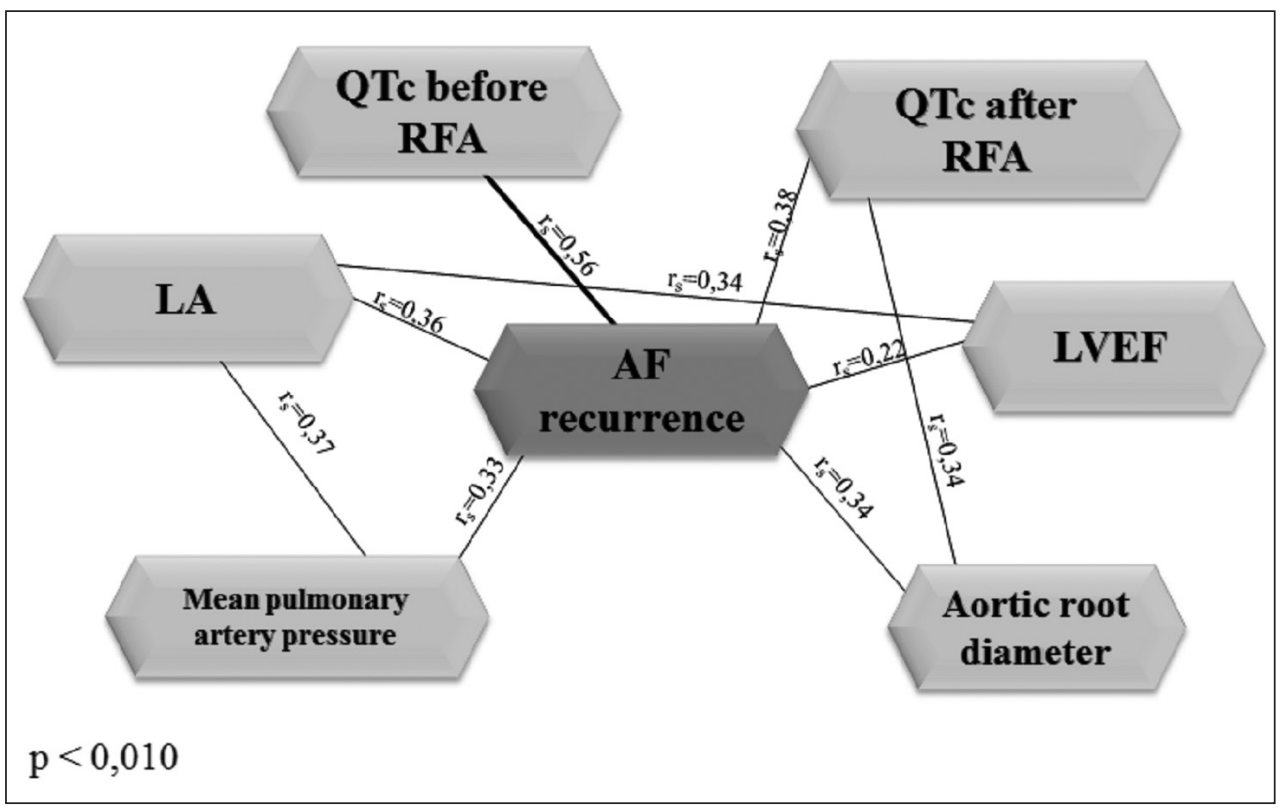

Fig. 2. Correlation analysis

LVED, LVES, LVEDV, LVESV, RA diameter of the two groups. HR and PR interval was comparable in both groups.

The AF recurrence group before RFA had a significantly longer QTc interval than the non-recurrence group $(387,23 \pm 2,31$ vs. $341,22 \pm 8,91 \mathrm{~ms},<0,010)$.
Figure 1 presents the significant difference in the QTc prolongation in both groups after RFA $(439,01 \pm 4,73$ vs. $373,21 \pm 7,92$ $\mathrm{ms}, \mathrm{p}<0,001$ for recurrence vs. non-recurrence group).

There was a correlation between AF recurrence and LA diameter, QTc before RFA, QTc after RFA, LVEF, aortic 

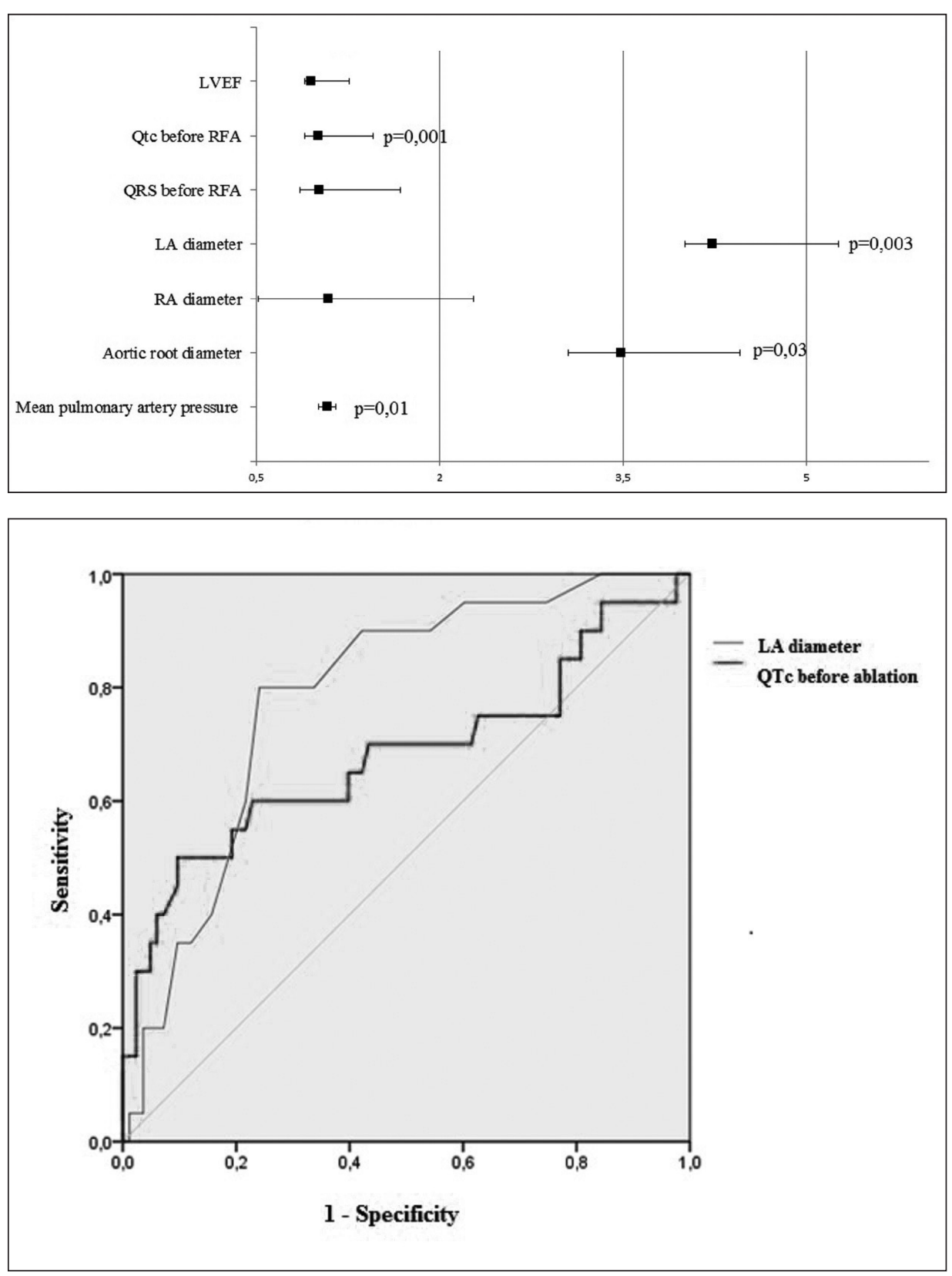

Fig. 3. Odds Ratios with 95\% Confidence Limits

Fig. 4. ROC Curve root diameter, mean pulmonary artery pressure $(\mathrm{p}$ for trend $<0,010$; Figure 2).

Figure 3 presents the odds ratios for LVEF, QTc before RFA, QRS before RFA, LA diameter, RA diameter, aortic root diameter, mean pulmonary artery pressure. The most significant are QTc before RFA, LA diameter, aortic root diameter and mean pulmonary artery pressure.

ROC curve analysis (Figure 4) revealed LA diameter the most sensitive factor for AF-recurrence after RFA.

The logistic regression model was done and the equation was created:

$\mathrm{R}^{2}=54,0 \%$

$\mathrm{Y}=22,6+\left(-2,47^{\star} \mathrm{X} 1\right)+\left(-2,1^{\star} \mathrm{X} 2\right)+\left(-0,02^{\star} \mathrm{X} 3\right)$

where $\mathrm{Y}$-outcome ( 1 - recurrence, 2 - no recurrence), $\mathrm{X}$-predictors in order of decreasing importance,

$\mathrm{X} 1$ - gender (1- male, 2- female),

$\mathrm{X} 2$ - LA diameter, $\mathrm{cm}$,
X3 - QTc before RFA, ms.

Model sensitivity $-50,0 \%$, specificity $-95,0 \%$.

\section{DISCUSSION}

The main findings of our study include that QTc prolongation before and after ablation was an independent risk factor for arrhythmia recurrence in patients with chronic HF who underwent RFA of the arrhythmia substrate. Patients with chronic HF with preserved LVEF and QTc before RFA more than 387 ms have a risk of recurrence of arrhythmia after intervention, so it is important to weigh carefully before deciding on a patient's AF strategy. There are few studies in the world that have studied QTc as a predictor of $\mathrm{AF}$ recurrence after ablation. $\mathrm{Ma}$ N. et al also showed the greater predicting value of combination of LA size and QTc duration in AF recurrence after catheter 
ablation. So, it was proposed to use these parameters to forecast the risk of AF recurrence in patients with type 2 diabetes mellitus undergoing catheter ablation [12].

The QT interval represents the ventricular repolarization and it is commonly supposed the presence of the correlation between atrial and ventricular repolarization. The data from during the 10-year period Danish registry showed a robust J-shaped association between QTc interval duration and the occurrence of AF [13]. The study of Liu N. also found the QTc interval prolongation was an independent risk factor for AF recurrence after RFA in patients with arterial hypertension, as well as LA diameter [14]. The limitations of these studies were: small cohorts of patients, the exclusion patients with chronic HF, especially with preserved LVEF.

Structural remodeling of AF is known to be associated with the risk of AF recurrence. The major factor of $\mathrm{AF}$ progression is atrial tissue fibrosis which affects the LA, hence, the more extensively altered LA the higher risk arrhythmia persistence. Postmortem histological analysis has shown a clear correlation between AF and the level of atrial fibrosis. The level of LA fibrosis is also the chief qualifier of RFA success for rhythm control strategy of treatment [15]. Our study is consistent with previous data that demonstrated an association between enlarged atria and AF recurrence after RFA compared to patients without recurrence. [16]. We found that patients with an enlarged LA diameter had a higher risk of AF recurrence after RFA of arrhythmia substrate. Thus, QTc duration provides additional information that can be used in conjunction with LA size to identify patients at high risk of AF recurrence.

The development of regression models for predicting a particular event is widely used in medicine. Our regression model is available to use and predict the risk of arrhythmia recurrence, as variables are easily identified in each patient. So far, no similar models have been developed. But regression analysis has its limitations. The low sensitivity of the model is due to probably insufficient number of studied factors or needs re-analysis at a longer observation period, but its high specificity has shown that the model can be further used for the group of patients with similar data hemodynamic and structural parameters of the heart with concomitant chronic HF with preserved LVEF.

\section{CONCLUSIONS}

1. QTc duration before radiofrequency ablation and its prolongation after intervention are independent predictors of atrial fibrillation recurrence in patients with chronic heart failure with preserved left ventricular ejection fraction.

2. Left atrium diameter before ablation is a highly sensitive predictor of atrial fibrillation recurrence in patients with chronic heart failure with a preserved left ventricular ejection fraction.

3. A developed regression model with sensitivity of $50,0 \%$ and specificity of $95,0 \%$ can be used to predict recurrence of arrhythmia after ablation in patients with chronic heart failure with preserved ejection fraction by using available parameters such as gender, left atrial diameter and QTc before ablation.

\section{REFERENCES}

1. Khan S., Rahman H., Talluri S., Kaluski E. The Clinical Benefits and Mortality Reduction Associated With Catheter Ablation in Subjects With Atrial Fibrillation. JACC: Clin Electrophysiol. 2018;4(5):626-635.

2. Shi L., Heng R., Liu S., Leng F. et al. Effect of catheter ablation versus antiarrhythmic drugs on atrial fibrillation: A meta-analysis of randomized controlled trials. Exp Ther Med. 2015;10(2):816-822.

3. Al Halabi S., Qintar M., Hussein A. et al. Catheter Ablation for Atrial Fibrillation in Heart Failure Patients: A Meta-Analysis of Randomized Controlled Trials. JACC Clin Electrophysiol. 2015;1(3):200-209. doi: 10.1016/j.jacep.2015.02.018.

4. Sultan A., Lüker J., Andresen D., Kuck K. etal. Predictors of Atrial Fibrillation Recurrence after Catheter Ablation: Data from the German Ablation Registry. Sci Rep. 2017;7(1). doi: 10.1038/s41598-017-16938-6.

5. Park J., Lee J., Yang P. et al. Good responders to catheter ablation for longstanding persistent atrial fibrillation: Clinical and genetic characteristics. J Cardiol. 2017;69(3):584-590.

6. Wen S., Liu N., Li S. et al. QTc Interval Prolongation Predicts Arrhythmia Recurrence After Catheter Ablation of Atrial Fibrillation in Patients With Hypertrophic Cardiomyopathy. Circ J. 2015;79(5):1024-1030.

7. Morttada D. Predictors of Short and Long Term Recurrences of Paroxysmal AF after Radiofrequency Ablation. Is Blanking Period Really Benign? J Atr Fibrillation. 2018;11(4):45.

8. Chou C., Lee H., Chang P. et al. Left atrial emptying fraction predicts recurrence of atrial fibrillation after radiofrequency catheter ablation. PLoS One. 2018;13(1):e0191196. doi: 10.1371/journal.pone.0191196.

9. Ponikowski P., Voors A.A., Anker S.D. et al. 2016 ESC Guidelines for the diagnosis and treatment of acute and chronic heart failure: The Task Force for the diagnosis and treatment of acute and chronic heart failure of the European Society of Cardiology (ESC). Developed with the special contribution of the Heart Failure Association (HFA) of the ESC. Eur J Heart Fail. 2016;37(27):2129-2200. doi: 10.1093/eurheartj/ehw128.

10. Lang R., Badano L., Mor-Avi V. et al. Recommendations for Cardiac Chamber Quantification by Echocardiography in Adults: An Update from the American Society of Echocardiography and the European Association of Cardiovascular Imaging. Eur Heart J Cardiovasc Imaging. 2015;16(3):233-271. doi: 10.1093/ehji/jev014.

11. Kirchhof P., Benussi S., Kotecha D. at al. ESC Scientific Document Group, 2016 ESC Guidelines for the management of atrial fibrillation developed in collaboration with EACTS, Eur Heart J. 2016;37(38): 2893-2962. doi:10.1093/eurheartj/ehw210.

12. Ma N., Wu X., Ma C. et al. QTC interval predicts outcome of catheter ablation in paroxysmal atrial fibrillation patients with type 2 diabetes mellitus. J Huazhong Univ Sci Technolog Med Sci. 2016;36(5):646-652.

13. Nielsen J., Graff C. et al. J-Shaped Association Between QTC Interval Duration and the Risk of Atrial Fibrillation. J Am Coll of Cardiol. 2013;61(25):2557-2564.

14. Liu N., Wen S., Ruan Y. et al. QTc interval prolongation predicts the ablation outcome in hypertensive patients with paroxysmal atrial fibrillation. Eur Heart JSupplements. 2015;17(B):B32-B38. doi: 10.1093/ eurheartj/suv017.

15. Marrouche N., Wilber D., Hindricks G. et al. Association of Atrial Tissue Fibrosis Identified by Delayed Enhancement MRI and Atrial Fibrillation Catheter Ablation. JAMA. 2014;311(5):498. 
16. Njoku A., Kannabhiran M., Arora R. et al. Left atrial volume predicts atrial fibrillation recurrence after radiofrequency ablation: a meta-analysis. Europace. 2017;20(1):33-42. doi: 10.1093/europace/eux013.

The research was carried out within the Scientific research work V.N. Karazin Kharkiv National University, Kharkiv, Ukraine; ID 0120 U101507 from 04.2020.

\section{ORCID and contributionship:}

Tetiana V. Zolotarova: 0000-0001-6226-6769 ${ }^{B, D}$

Mariia S. Brynza: 0000-0002-8260-3600 C

Dmytro Y. Volkov: 0000-0002-3690-1308 ${ }^{E}$

Maryna I. Shevchuk: 0000-0002-7632-893X ${ }^{E}$

Oleksandr V. Bilchenko: 0000-0003-3313-2547 A,F

\section{Conflict of interest:}

The Authors declare no conflict of interest.

\section{CORRESPONDING AUTHOR}

Tetiana V. Zolotarova

V. N. Karazin Kharkiv National University

4 Svobody Sq., 61022 Kharkiv, Ukraine

tel: +380502140828

e-mail: tatiana.zolotarova@karazin.ua

Received: 02.07 .2020

Accepted: 02.06 .2021

A - Work concept and design, B - Data collection and analysis, C - Responsibility for statistical analysis,

D -Writing the article, $\mathbf{E}$-Critical review, $\mathbf{F}$ - Final approval of the article 